\title{
Implementing accessibility settings for people living with dementia in touchscreen apps
}

Article

Accepted Version

Joddrell, P. and Astell, A. J. (2019) Implementing accessibility settings for people living with dementia in touchscreen apps. Gerontology, 65 (5). pp. 560-570. ISSN 0304-324X doi: https://doi.org/10.1159/000498885 Available at https://centaur.reading.ac.uk/82120/

It is advisable to refer to the publisher's version if you intend to cite from the work. See Guidance on citing.

To link to this article DOI: http://dx.doi.org/10.1159/000498885

Publisher: Karger

All outputs in CentAUR are protected by Intellectual Property Rights law, including copyright law. Copyright and IPR is retained by the creators or other copyright holders. Terms and conditions for use of this material are defined in the End User Agreement.

www.reading.ac.uk/centaur

\section{CentAUR}


Central Archive at the University of Reading

Reading's research outputs online 
TITLE: Implementing accessibility settings for people living with dementia in touchscreen apps

Dr Phil Joddrell (corresponding author)

The University of Sheffield, Centre for Assistive Technology and Connected Healthcare, School of Health and Related Research, 217 Portobello, Sheffield, S1 4DP, UK p.joddrell@sheffield.ac.uk, +441142220779

Professor Arlene J. Astell

${ }^{2}$ University of Reading, School of Psychology \& Clinical Language Sciences, Earley, Reading RG6 6BZ, UK

${ }^{3}$ Department of Occupational Therapy \& Occupational Sciences and Department of Psychiatry, University of Toronto, Canada

${ }^{4}$ Ontario Shores Centre for Mental Health Sciences, Whitby, Canada a.astell@ reading.ac.uk, +441183788393

Declarations of interest: none 


\section{Abstract}

INTRODUCTION: Accessibility options within apps can enable customisation and improve usability. This study set out to examine whether the introduction of accessibility settings for people with dementia in touchscreen apps could improve the user experience.

METHODS: Thirty participants were recruited to play on three occasions, one of two apps adapted with the inclusion of accessibility features derived from an analysis of gameplay in a previous study. The results were compared with those from the earlier study (i.e. pre-adapted apps).

RESULTS: The accessibility features significantly improved usability in the previously more difficult app (Solitaire) and maintained playability with marginal improvements in the previously more accessible app (Bubble Explode).

DISCUSSION: This first study of implementing accessibility settings for dementia in mainstream apps demonstrate their feasibility and impact. The findings reveal core principles of touchscreen interaction and design for dementia that can inform future app development.

\section{Keywords}

Technology; Accessibility; Leisure 


\section{Introduction}

Accessibility is a key concept of interactive systems that promotes equal opportunities for all users [1]. Within digital applications (apps), settings menus are commonly used to present accessibility options, enabling the appearance and sounds of the app to be customised to suit the user requirements [2]. Some apps include specific accessibility settings to address the needs of people living with a particular condition, e.g. autism [3] or aphasia [4]. Whilst website accessibility for people living with dementia has received some attention $[5,6]$, there has been no exploration of accessibility settings in apps for this population. Given the omnipresence of apps on technologies such as smartphones and tablet computers, there is a need to explore accessibility settings for people living with dementia to prevent digital exclusion. Adapting existing apps used by the wider population presents a vast array of choice as well as a route to reducing stigma [7]

An initial study conducted in 2015, demonstrated that people with dementia could independently initiate and engage with selected apps [8]. This first phase focused on gaming apps, as part of a programme addressing the need for independent leisure activities for people with dementia [9-11]. Thirty people with dementia tested two apps - Solitaire, a traditional card game, and Bubble Explode, a tile matching game - on three occasions. These games were selected based on an evaluation process that identified a wide range of generic accessibility options in both apps [8].

Phase 1 reported that $90 \%$ of participants independently initiated gameplay, with $88 \%$ enjoying playing the games. However, the two games differed in playability with $93 \%$ of participants reaching a predetermined checkpoint in Bubble Explode compared with only 17\% playing Solitaire. Our analysis of the gameplay identified issues in both apps relating to accessibility that disrupted gameplay for many of the participants, although this was more apparent in Solitaire. For example, Solitaire, has two possible control methods: (i) 'drag and drop', where 
the user touches the card they want to move and slides their finger to the desired location to place it, or (ii) 'tap', where the user simply touches the card they want to move and the computer automatically places it if there is a viable placement. The concurrent presence of both control methods created an accessibility problem as the computer sometimes misinterpreted the user's intention, either by moving the card automatically if the user raised their finger from the screen during a 'drag and drop' attempt, or by not moving the card automatically if the user held their finger down too long during a 'tap' move. This is especially problematic for users with dementia who are at increased risk of being confused when the game does not behave as expected.

These and other disruptions identified during app usage in phase one, highlighted the need for accessibility settings designed specifically for people living with dementia. We discussed the problems associated with each game with the respective app developers, and collaboratively agreed adaptations to improve accessibility (see section 2.3). To evaluate the effectiveness of these adaptations, we designed the current study (phase 2) replicating the methods and experimental design employed in phase 1 with the adapted apps [8]. The following research question was addressed: Can the implementation of tailored settings improve the accessibility of existing touchscreen apps for people living with dementia?

\section{Method}

This study replicates the method used in phase 1 , a detailed description of which can be found here [8]. 


\subsection{Design}

All conditions were replicated from phase 1, with the exception of the apps being adapted with dementia-specific accessibility settings. Two new groups of 15 participants were recruited, to play the updated versions of the apps on three separate occasions.

\subsection{Participants}

The project received a favourable ethical opinion from the School of Health and Related Research (ScHARR) Ethics Committee at The University of Sheffield. A member of the research team obtained consent from each participant. Thirty people living with dementia were recruited from residential and specialist dementia services in Sheffield, UK. Twenty-two of the participants were female and eight were male. Their mean age was 84.17 years (range 66-102; SD 8.35). The severity of their cognitive impairment was assessed using the Montreal Cognitive Assessment (MoCA [12]), with a score of <26/30 required to distinguish between dementia and healthy controls. The participants' mean score on the MoCA was 12.97 (range 4-24; SD 4.9).

Of the 30 participants recruited to phase 2, 26 engaged at all three time-points and four engaged at two time-points. This resulted in a total of 86 sessions out of a possible 90 . The missing data were accounted for by: participants missing a session through ill health (two occasions); participants being judged to having shown signs of discomfort at a previous session (one occasion); or participants declining to participate on the day of the session (one occasion). Due to equipment failure, the video recordings of two gameplay sessions could not be analysed. Therefore, the results relate to 84 recorded gameplay sessions (43 for Solitaire and 41 for Bubble Explode). In comparison with phase 1, there were five more sessions attended by participants playing Solitaire in the present phase, but the same number of sessions attended involving Bubble Explode. 


\subsection{Materials}

To improve accessibility, the problems associated with each app were discussed with the respective developers, and design adaptations were agreed collaboratively (see Table 1). For Solitaire, once the collaborative discussion phase with the developers was completed, the three agreed adaptations were all implemented as expected in the app update. However, with Bubble Explode, of the four agreed adaptations, three were only partially implemented and the other was a compromised solution. Updates for both apps including the adaptations were released within nine months.

An Apple iPad (fourth generation) running iOS 9 was used for all participants playing Solitaire, and a Samsung Galaxy Tab (S2) running Android 7.0 (Nougat) was used for all participants playing Bubble Explode. Both tablets were presented in a 'Proud to Play' purpose-designed case for people living with dementia, created as part of the international 'InTouch' research project [13]. A Panasonic HD digital video recorder (model HC-X900) on a tripod was used to record all data collection sessions.

\subsection{Procedure}

The sessions were conducted in a suitable environment within each care service that ensured privacy and comfort. The video camera was positioned on a tripod in a position allowing a view of the tablet screen over the participant's shoulder.

For each participant the following procedure was used at each data collection session. The tablet was presented to the participant with the start of the game ready on the screen. The researcher provided a rehearsed physical demonstration of the game, in combination with verbal instructions describing the process. The researcher then reset the game to the beginning 
and invited the participant to begin in his or her own time. Participants were given the opportunity to play the game through to completion unless they indicated that they wanted to finish earlier or if their gameplay session exceeded 10 minutes. As the focus of the research was on independent gaming, the researcher retreated out of the participant's line of sight and resisted any initial requests for advice or support from the participant during gameplay by politely encouraging them to try and continue themselves. However, if the participant requested support more than twice, or was deemed to be in any discomfort or distress, the researcher responded to the participant and offered support, thus ending their gameplay session for the purpose of analysis.

\subsection{Video coding}

After all data had been collected, each video recorded gameplay session was analysed using the coding scheme presented in Table 2. Analysis was conducted using The Observer® XT (version 12.0.825) software by Noldus Information Technology on a Dell Precision T3610 computer running Windows 7 Professional.

\subsection{Outcome measures}

Accessibility and gameplay were measured through analysis of the coded video data.

\subsubsection{Accessibility}

Three outcomes were measured to assess the effectiveness of the accessibility settings (see Table 2).

\section{Game advancing moves.}

The percentage of screen interactions coded as advancing the gameplay was calculated from the total number of intentional screen interactions in each gameplay session. In Solitaire, game 
advancing moves were defined as drawing cards from the deck or placing cards in viable locations, and in Bubble Explode as removing coloured groups of bubbles.

\section{Usability problems.}

The percentage of screen interactions that were coded as being indicative of an issue relating to usability was calculated from the total number of screen interactions in each gameplay session. Usability problems for both apps were defined as attempted but unsuccessful viable moves, unintentional screen interactions or interactions with on-screen elements not directly related to gameplay (e.g., menu icons).

\section{Utilised prompts.}

The percentage of prompts to which participants responded was calculated from the total number of displayed prompts in each gameplay session. This included the inactivity prompts found in both apps, as well as the redirection prompt following an invalid move attempt in Bubble Explode. Utilising a prompt was defined as attempting the highlighted move as the next screen touch.

\subsubsection{Independent gameplay and enjoyment}

With the implementation of new accessibility features designed to improve the gameplay experience for people living with dementia, it was important to repeat the original outcome measures [8] to investigate the impact of the adaptations. Therefore, the following variables were measured through the video coding process (see Table 2), for comparison with phase 1 .

\section{Independent gameplay initiation.}

Participants were observed for independent initiation of gameplay, once the rules had been explained to them and they were invited to start.

\section{Checkpoint attainment.}


Participants were observed for independent advancement through the game to a pre-determined 'checkpoint' [8].

\section{Enjoyment.}

Participants were asked whether or not they had enjoyed their experience at the end of each gameplay session.

\subsection{Data analysis}

The coded data were analysed using appropriate statistical analyses (independent samples $t$ tests, chi-square tests for homogeneity, Fischer's exact tests).

\section{Results}

To assess the effectiveness of the implemented adaptations for both Solitaire and Bubble Explode, the data are compared with the equivalent data from phase 1. Participant characteristics from both phases are presented in Table 3. There was no significant difference between the age of the participants in phase $1(M=87.33, S E=0.97)$ and phase $2(M=84.17$, $S E=1.52 ; t(58)=1.75, p=.09, r=.22)$, and no significant difference between their MoCA scores in phase $1(M=13.4, S E=0.55)$ and phase $2(M=12.97, S E=0.9 ; t(48.06)=0.41, p$ $=.68, r=.06)$. None of the participants recruited to either phase reported having had any experience using tablet computers prior to this research project.

Table 4 presents the total counts of all screen interactions made by participants compared between phases 1 and 2. The outcomes related to accessibility for both phases and both apps are derived from the figures in this table, calculated as proportions according to the definitions described in section 2.5. 


\subsection{Solitaire (Group 1)}

Comparisons of accessibility and gameplay (Table 5) were conducted between the original and adapted versions of Solitaire. The proportion of game advancing moves in the adapted version $(M=50.1, S E=6.36)$ did not differ significantly to the original version $(M=36.45, S E=8)$. However, usability problems were significantly reduced in the adapted Solitaire $(M=12.65, S E$ $=2.41)$ compared with the original version $(M=44.05, S E=5.48)$. There was also a significant increase in the proportion of prompts utilised in the adapted version $(M=36.41, S E=7.32)$ compared with the original version $(M=15.01, S E=7.33$; Table 5).

In terms of gameplay, there was a significant increase in independent initiation in the adapted version of Solitaire compared to the original (Table 5). There was no significant change in independent advancement to the checkpoint and enjoyment was not significantly changed.

\subsection{Bubble Explode (Group 2)}

Accessibility and gameplay (Table 5) were compared between the original and adapted versions of Bubble Explode. There was no significant difference in the proportion of game advancing moves between the adapted version $(M=69.85, S E=4.28)$ and the original version $(M=69.36, S E=4.32)$, and usability problems remained low in both the adapted version $(M$ $=9.3, S E=2.06)$ and the original version $(M=8.29, S E=1.66)$. As the prompt feature was newly introduced for the adapted version of Bubble Explode, there is no comparative data from phase 1. Descriptive statistics reveal that just over $10 \%$ of the prompts that appeared on screen were utilised by participants (see Table 7). This figure is lower than for both designs in the original (20\%) and adapted (61\%) versions of Solitaire (Table 5).

Independent initiation of gameplay remained at ceiling level (100\%) for the adapted Bubble Explode, and there were marginal but non-significant increases in both independent advancement and game enjoyment (Table 5). 


\section{Discussion}

This study examined the implementation of accessibility features designed for people with dementia into two mainstream apps. The first of these, Solitaire, was originally difficult for people to play despite the presence of accessible features such as changing the colours of the game backgrounds, the face of the cards and a next move prompt feature [9]. The adapted version of Solitaire with new accessibility features significantly increased independent initiation of gameplay and reduced the number of usability problems experienced by people with dementia. In addition, redesigning the prompt feature significantly increased its utilisation during gameplay. This suggests that the adaptations - a simplified control method, a less disruptive method of activating the toolbar and a redesigned prompt feature (Table 2) - were effective in improving the accessibility of the app for people living with dementia; removing or at least minimising the barriers identified in phase 1. Further examination of the various types of usability problems (unsuccessful moves, unintentional touches and non-game interactions) revealed that the total count of each substantially decreased (see Table 4) in comparison with the results from phase 1 , despite there being more initiated gameplay sessions and therefore more overall touches. This is important because several of the individual barriers identified from the data in phase 1 were attributed to specific categories of touch. Consequently, whilst the overall reduction in usability problems indicates improved accessibility generally, the finding that all three of these categories decreased provides evidence that the individual adaptations were effective.

In phase 1 the original Bubble Explode proved to be an accessible game with independent gameplay initiated in $100 \%$ of sessions. In contrast to Solitaire, Bubble Explode is a less complex game, with a single control method - tap - and simpler rules - remove same colour blocks of bubbles. Usability problems in Bubble Explode were low in both the original (7.8\%) and the adapted version (7.6\%). Interestingly, utilisation of the new prompt feature in the 
adapted Bubble Explode was low, with just $10 \%$ of all generated prompts being utilised, even though a prompt was identified as something that could be helpful in the gameplay analysis of phase 1. Two possible explanations for the lower impact of the Bubble Explode adaptations are considered. Firstly, the original Bubble Explode was already a highly accessible game, and it is possible that marginal improvements were all that could have been realistically achieved. However, many of the identified problems in phase 1 (see Table 1), on which the implemented app adaptations were based, were again observed in the present phase. Consequently, the second explanation proposed is that the adaptations that were actually implemented were less consistent with what was proposed as solutions based on the gameplay analysis. For example, the newly introduced prompt feature was very subtle, and there was no audible or animated feedback assigned to an invalid move attempt (although this was requested).

The ability to customise software has been highlighted as a key benefit of modern touchscreen devices for people with dementia [14]. The adaptations to Solitaire were all included as customisation options within the existing app (see 1.1), to allow users to select which of them, if any, they wish to apply during gameplay. Whilst the Bubble Explode developers did not include the adaptations as options, instead implementing them as design changes for all app users, they still adapted their existing app, as opposed to releasing a separate version specifically for dementia. By including adaptations and customisation options for dementia in this format, a blueprint has been laid out that other developers can follow. To our knowledge, these are the first examples of accessibility options specifically designed for people with dementia to be incorporated into mainstream apps (see Figure 1). The benefit to increasing the accessibility of existing apps is that people can tailor the gameplay experience to fit their own needs. Dementia affects each individual uniquely [15], and therefore no combination of settings will suit everybody. However, by including adaptations as a series of options that can be turned on or off, the accessibility of apps can impact a wider audience. 
A further benefit to the incorporation of accessibility settings in existing apps relates to the stigmatisation that can arise through the design of technology that is set apart from other products by its association with disability [16]. A separately-released 'Bubble Explode for Dementia', for example, would be unnecessarily segregated from the original game based on just a few accessibility features that allow the game to be played by a wider audience. By offering all apps together, people with dementia are able to share the same technology-use experience without risking isolation. This has the potential to encourage intergenerational socialisation and raise awareness of dementia with younger audiences [17].

Finally, whilst the participants in the present project reported having no prior tablet computer experience, it is inevitable that people receiving diagnoses of dementia now, and increasingly in the future, will be existing users. By 2020 , it is forecast that 1.4 billion people globally will be tablet users [18]. Whilst focused on gaming apps, the results of this research reveal core principles relating to accessibility for dementia, both in terms of how people interact with apps and devices, and the optimum design of content, which can be generalised to other types of apps (e.g. finance, health management, etc.). If the implementation of accessibility options for people with dementia were to be widely adopted by app developers, existing app users who receive a diagnosis of dementia would have an increased opportunity of continuing to use the same software while only having to adjust the settings to meet their changing needs. This corresponds with continuity theory [19], which emphasises the crucial role that continuity of activity can have on preserving a sense of identity and self-concept, and has also been linked to improved self-esteem [20].

\subsection{Conclusion}

Incorporating tailored accessible design within existing apps can improve the experience of using tablet computers for people living with dementia. This highlights the potential of apps to 
provide opportunities for leisure and engaging activity for people with dementia, just like for the rest of the population. This research demonstrates how the specific needs of this population can be conveyed to app developers to incorporate accessibility features for dementia. 


\section{Solitaire (MobilityWare)}

\section{Identified problems}

Two user control methods ('drag and drop'

and 'tap') functioning concurrently

Pop-up toolbar that was frequently triggered

unintentionally

Auto-prompt feature which proved

ineffective during gameplay

\section{Collaboratively agreed solutions}

Added option to select one control method

from the menu*

Added option to change the input method

required to trigger the toolbar*

Added option to emphasise the visual

presentation of the auto-prompt*

Bubble Explode (Spooky House Studios)

\section{Identified problems}

Overlay of menu buttons and interactive

elements at the start of gameplay

Text feedback, in addition to other forms of

feedback, that proved distracting

No auto-prompt feature if users are inactive

No feedback given for incorrect moves

\section{Collaboratively agreed solutions}

Adapted layout of opening gameplay screen

Adapted presentation of text feedback

Inclusion of auto-prompt feature for inactivity

Assign audio and visual feedback to an

incorrect move attempt 
Table 2. Summary of coding scheme designed for the purposes of this research project to observe all user-led screen interactions and the presence of certain app features

\section{Screen \\ Definition}

interactions

Game advancing

An intentional game move that is valid and successfully completed move

\section{Unsuccessful}

An intentional game move that is valid but not successfully completed move

Invalid move

An intentional game move that is invalid (i.e., does not comply with the rules of the game)

\begin{tabular}{ll}
\hline Unintentional & An interaction with the screen that was not intended by the participant \\
interaction & An interaction with the screen that in intentional but not directly related to the \\
\hline Non-game & game (i.e., a menu item)
\end{tabular}

\section{Gameplay Definition}

Gameplay

initiated

Player begins gameplay (first screen interaction after demonstration)

Checkpoint

reached

Checkpoint of the game is reached independently by the player

Checkpoint not Checkpoint of the game is not reached by the player
reached

\begin{tabular}{ll}
\hline Prompts & Definition \\
\hline No prompt & No prompt is displayed on the screen \\
\hline Prompt & Prompt is displayed on the screen
\end{tabular}


Prompt utilised Next intentional screen interaction attempts highlighted move

Prompt not utilised

Next intentional screen interaction does not attempt highlighted move 
Table 3. Characteristics of participants in phases 1 and 2

\begin{tabular}{|c|c|c|c|c|c|c|}
\hline & & Female & Male & $\begin{array}{l}\text { Mean age } \\
(\mathrm{SD})\end{array}$ & $\begin{array}{l}\text { Mean MoCA } \\
\text { score /30 } \\
\text { (SD) }\end{array}$ & $\begin{array}{l}\text { Total no. } \\
\text { of } \\
\text { sessions }\end{array}$ \\
\hline Solitaire & Phase 1 & 12 & 3 & $87.53(5.89)$ & $13.07(2.84)$ & 38 \\
\hline (Group 1) & Phase 2 & 13 & 2 & $85.4(6.61)$ & $12.8(4.78)$ & 43 \\
\hline Bubble Ex. & Phase 1 & 13 & 2 & $87.13(4.93)$ & $13.73(3.22)$ & 43 \\
\hline (Group 2) & Phase 2 & 9 & 6 & $82.93(9.87)$ & $13.13(5.18)$ & 43 \\
\hline
\end{tabular}


Table 4. Total counts of screen interactions in original and adapted versions of both apps where gameplay was initiated

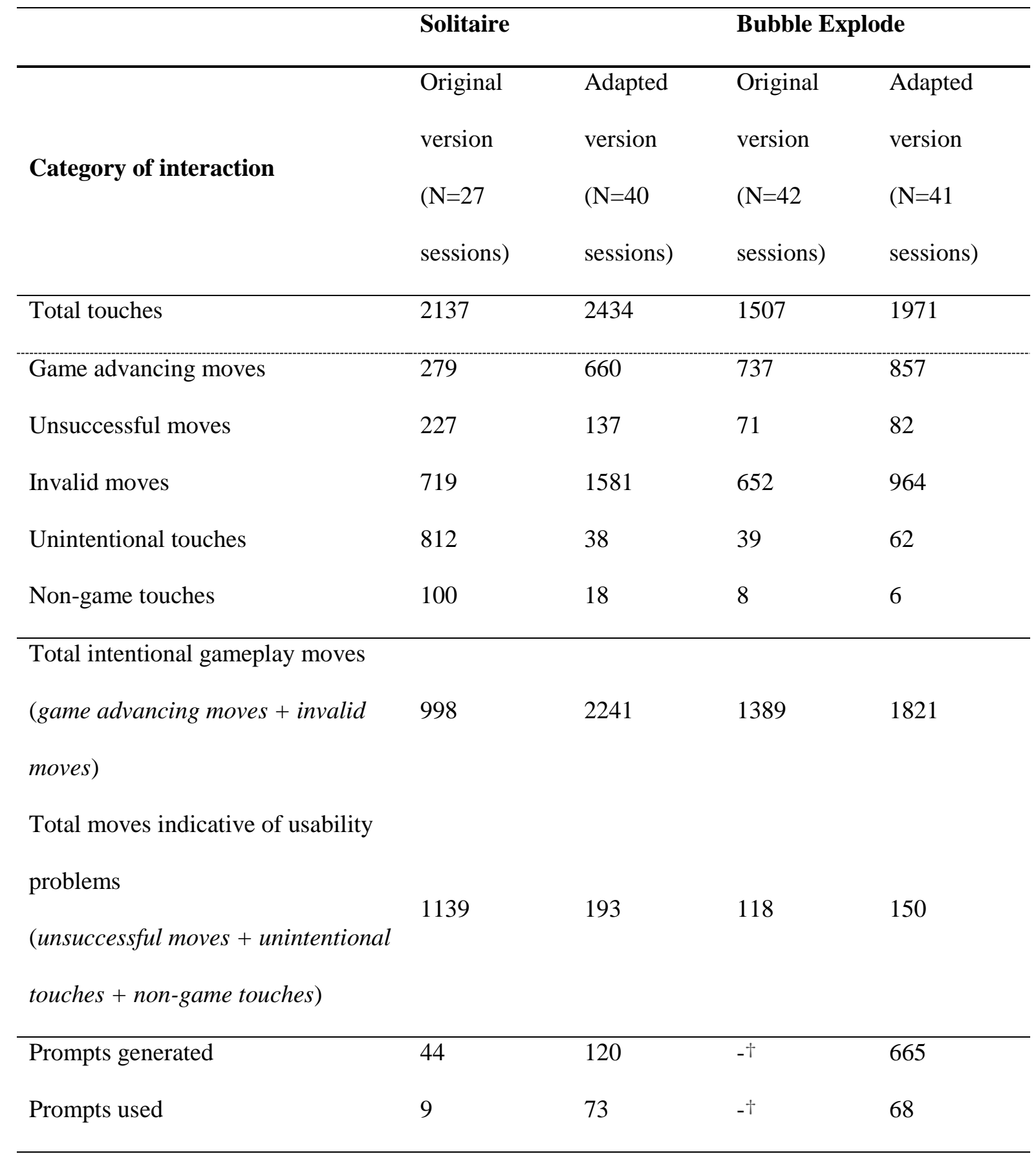

tNew feature not present in original version of the app 
Table 5. Summarised outcomes relating to accessibility, independent gameplay and enjoyment from gameplay sessions involving both original and adapted versions of both apps 


\section{Total (\%)}

\section{Outcome}

Original version Adapted version

( $\mathrm{N}=27$ sessions $) \quad(\mathrm{N}=40$ sessions $)$

Test of independence Sig.

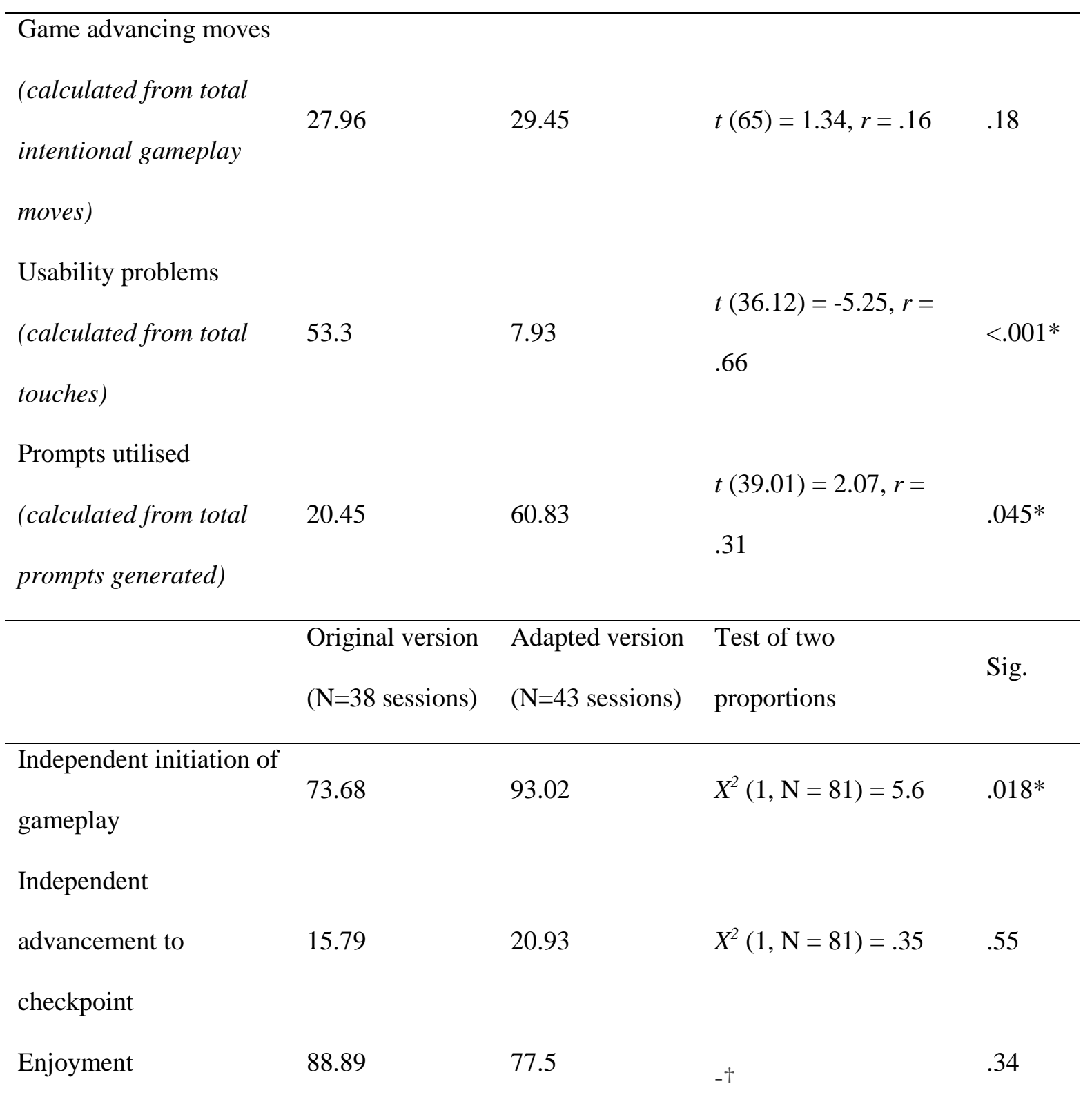

\section{Bubble Explode}

\section{Total (\%)}

Outcome

Original version Adapted version

( $\mathrm{N}=42$ sessions $) \quad(\mathrm{N}=41$ sessions $)$

Test of independence Sig. 


\begin{tabular}{|c|c|c|c|c|}
\hline \multicolumn{5}{|l|}{ Game advancino moves } \\
\hline $\begin{array}{l}\text { (calculated from total } \\
\text { intentional gameplay }\end{array}$ & 53.06 & 47.06 & $t(81)=.08, r=.01$ & .94 \\
\hline \multicolumn{5}{|l|}{ moves) } \\
\hline \multicolumn{5}{|l|}{ Usability problems } \\
\hline (calculated from total & 7.83 & 7.61 & $t(81)=.38, r=.04$ & .71 \\
\hline \multicolumn{5}{|l|}{ touches) } \\
\hline \multicolumn{5}{|l|}{ Prompts utilised $\neq$} \\
\hline (calculated from total & - & 10.23 & - & - \\
\hline \multicolumn{5}{|l|}{ prompts generated) } \\
\hline & $\begin{array}{l}\text { Original version } \\
(\mathrm{N}=43 \text { sessions })\end{array}$ & $\begin{array}{l}\text { Adapted version } \\
\text { (N=41 sessions) }\end{array}$ & $\begin{array}{l}\text { Test of two } \\
\text { proportions }\end{array}$ & Sig. \\
\hline $\begin{array}{l}\text { Independent initiation of } \\
\text { gameplay }\end{array}$ & 100 & 100 & N/A & N/A \\
\hline Independent & & & & \\
\hline advancement to & 76.74 & 87.8 & $X^{2}(1, \mathrm{~N}=84)=1.75$ & .19 \\
\hline checkpoint & & & & \\
\hline Enjoyment & 83.72 & 95.35 & $-\dagger$ & .16 \\
\hline
\end{tabular}

$*<.05$ significance, ${ }^{\dagger}$ Due to small sample sizes, Fisher's exact test was used, $¥$ New feature not present in original version of the app 


\section{Acknowledgements}

This research was conducted as part of the first author's doctoral thesis funded by the Centre for Assistive Technology and Connected Healthcare (CATCH) at the University of Sheffield. The work of the second author is supported by the Ontario Shores Foundation through the Research Chair in Dementia Wellbeing at the University of Toronto. 


\section{References}

[1] Gulliksen J, Harker S. The software accessibility of human-computer interfaces - ISO technical specification 16071. Univers Access Inf Soc 2004;3:6-16. doi:10.1007/s10209-003-0079-1.

[2] Richards JT, Hanson VL. Web accessibility: A broader view. Proc. 13th Int. Conf. World Wide Web, New York, New York, USA: ACM Press; 2004, p. 72. doi:10.1145/988672.988683.

[3] Leijdekkers P, Gay V, Wong F. CaptureMyEmotion: A mobile app to improve emotion learning for autistic children using sensors. Proc. CBMS 2013 - 26th IEEE Int. Symp. Comput. Med. Syst., IEEE; 2013, p. 381-4. doi:10.1109/CBMS.2013.6627821.

[4] Brandenburg C, Worrall L, Rodriguez AD, Copland D. Mobile computing technology and aphasia: An integrated review of accessibility and potential uses. Aphasiology 2013;27:444-61. doi:10.1080/02687038.2013.772293.

[5] Freeman E, Clare L, Savitch N, Royan L, Litherland R, Lindsay M. Improving website accessibility for people with early-stage dementia: A preliminary investigation. Aging Ment Heal 2005;9:442-8. doi:10.1080/13607860500142838.

[6] Savitch N, Zaphiris P, Clare L, Freeman E. Learning from people with dementia to improve accessibility of website interfaces. Br. HCl Conf., Citeseer; 2004.

[7] Smith SK, Mountain GA. New forms of information and communication technology (ICT) and the potential to facilitate social and leisure activity for people living with dementia. Int J Comput Healthc 2012;1:332. doi:10.1504/IJCIH.2012.051810.

[8] Astell AJ, Joddrell P, Groenewoud H, de Lange J, Goumans M, Cordia A, et al. Does familiarity affect the enjoyment of touchscreen games for people with dementia? Int 
J Med Inform 2016;91:e1-8. doi:10.1016/j.ijmedinf.2016.02.001.

[9] Schikhof Y, Groenewoud JH, Cordia AL, Lange J De. Designing happy games (apps) for people with dementia. Gerontechnology 2014;13:160-1.

doi:10.4017/gt.2014.13.02.106.00.

[10] Groenewoud H, de Lange J, Schikhof Y, Astell AJ, Joddrell P, Goumans M. Experiences of people with dementia playing casual games on a tablet. Gerontechnology 2017;16:37-47. doi:10.4017/gt.2017.16.1.004.00.

[11] Joddrell P, Hernandez A, Astell AJ. Identifying existing, accessible touchscreen games for people living with dementia. In: Miesenberger K, Buhler C, Penaz P, editors. Lect. Notes Comput. Sci., Berlin: Springer International Publishing; 2016, p. 509-14. doi:10.1007/978-3-319-41264-1_69.

[12] Nasreddine ZS, Phillips NA, Bédirian V, Charbonneau S, Whitehead V, Collin I, et al. The Montreal Cognitive Assessment, MoCA: A brief screening tool for mild cognitive impairment. J Am Geriatr Soc 2005;53:695-9. doi:10.1111/j.1532-5415.2005.53221.x.

[13] Cordia AL. Design of an iPad cover for people with cognitive impairment. Gerontechnology 2015;13:426-7. doi:10.4017/gt.2015.13.4.010.00.

[14] Joddrell P, Astell AJ. Studies involving people with dementia and touchscreen technology: A literature review. JMIR Rehabil Assist Technol 2016;3:e10. doi:10.2196/rehab.5788.

[15] Werner P, Savva GM, Maidment I. Dementia: Introduction, epidemiology and economic impact. Ment. Heal. Older People A Guid. Prim. Care Pract., Cham: Springer International Publishing; 2016, p. 197-209. doi:10.1007/978-3-319-29492-6.

[16] Rosenberg L, Kottorp A, Nygård L. Readiness for technology use with people with dementia: The perspectives of significant others. J Appl Gerontol 2012;31:510-30. 
doi:10.1177/0733464810396873.

[17] Cutler C, Hicks B, Innes A. Does digital gaming enable healthy aging for communitydwelling people with dementia? Games Cult 2016;11:104-29.

doi:10.1177/1555412015600580.

[18] Statista. Number of tablet users worldwide from 2013 to 2020 (in billions). Statista 2015. https://www.statista.com/statistics/377977/tablet-users-worldwide-forecast (WebCite Cache ID 6tdbuqQzW) (accessed September 21, 2017).

[19] Morgan-Brown M, Brangan J. Capturing interactive occupation and social engagement in a residential dementia and mental health setting using quantitative and narrative data. Geriatrics 2016;1:15. doi:10.3390/geriatrics1030015.

[20] Boyd HC, Evans NM, Orpwood RD, Harris ND. Using simple technology to prompt multistep tasks in the home for people with dementia: An exploratory study comparing prompting formats. Dementia-International J Soc Res Pract 2017;16:42442. doi:10.1177/1471301215602417. 